\title{
Thickened and Paste Tailings Pipeline Systems: Design Procedure - Part 1
}

\author{
R. Cooke Paterson and Cooke Consulting Engineers (Pty) Ltd, South Africa
}

\section{INTRODUCTION}

The design methodology for pipeline systems conveying thickened and paste tailings systems has been well developed over the last decade. This series of papers outlines the process for designing and implementing a typical surface tailings or underground backfill pipeline system. The papers comprise the following parts:

- Part 1 (this paper) discusses:

○ development of the design criteria document,

o issues to be considered for the test work, and

o pipeline flow behaviour modelling, friction loss calculation and pipe diameter selection.

- Part 2, to be presented at Paste and Thickened Tailings 2007, will include:

○ centrifugal pump performance derating,

○ hydraulic and mechanical design,

○ operational and control considerations, and

- specific considerations for thickener underflow and gravity flow systems.

\section{$1.1 \quad$ Terminology}

Our company classifies tailings and backfills according to the following criteria:

- The upper limit for conventional tailings is considered to correspond to the freely settled packing concentration. This typically corresponds to yield stresses of between 5 and $20 \mathrm{~Pa}$.

- High concentration tailings or thickened tailings is considered to cover the range from the freely settled concentration to the concentration at which the mixture has a fully sheared yield stress corresponding to $100 \mathrm{~Pa}$. Figure 1 illustrates the slump of a mixture with a $100 \mathrm{~Pa}$ yield stress.

- Paste tailings and fill are considered to be mixtures with yield stresses greater than $100 \mathrm{~Pa}$. The practical upper limit for pipeline transport is about $800 \mathrm{~Pa}$.

These mixtures may be transported in turbulent or laminar flow: 
- Turbulent flow - inertial forces dominate and the friction losses are relatively insensitive to the tailings rheology.

- Laminar flow - viscous forces dominate and the friction losses are directly related to the tailings rheology, which in turn is strongly effected by the tailings material properties, water chemistry and solids concentration.

- The transition zone from laminar flow occurs over a range of pipeline flow rates and is characterised by fluctuating pressure gradients.

Thickened tailings are typically transported in laminar flow, but turbulent flow operation is possible for low yield stress mixtures, large diameter pipes and high operating velocities. Paste tailings are always transported in laminar flow.

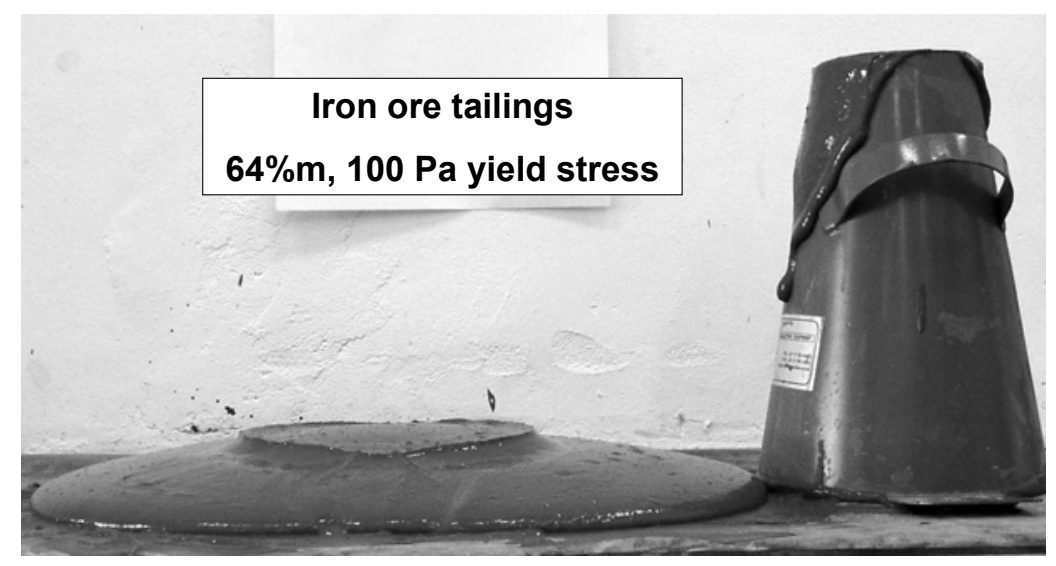

\section{Figure 1 Slump at transition from high concentration tailings to paste}

\subsection{Design process}

The procedure outlined in this paper is a guideline defining the typical steps to be followed when designing a pipeline system transporting thickened tailings or paste. It is not a definitive procedure that can be followed without a suitable background in the field and a proper understanding of thickened and paste tailings flow behaviour.

It is also important to note that the design process is iterative in nature. So while the steps have been laid out in an ideal linear path, the reality is that the process will be more chaotic with frequent jumps between the various steps of the process.

\section{DESIGN CRITERIA DOCUMENT}

The design criteria is the first formal document developed during the design process. Initially the document primarily specifies the system duty and the physical site conditions. As the design develops, the document expands to include the output of test work, design calculations and trade-off studies. It is a key document 
defining the client's specified requirements for the system. The client should approve each issue of the document before work proceeds on the system.

The format of the design document varies, but will generally include the sections discussed below.

\subsection{General}

The function of the system is defined with a basic description of the process. This section also includes:

- The design battery limits.

- Units and abbreviations to be used in the design.

- Reference documents.

- Site and environmental conditions. The site elevation and temperature is required to determine derating factors for electric motors. The site elevation also establishes the medium vapour pressure for hydraulic design calculations. The temperature range and seismic conditions are required for the mechanical design of the pipeline system.

\subsection{Process requirements}

This section defines the process requirements for the system:

- Medium description, design tonnages, concentrations, surge allowances and operating hours.

- Design life. This is required for determining life-cycle costs during trade-off studies. The design life is also an important consideration when specifying the pipeline materials - ideally the pipeline should last for the system life.

- The required bund capacity for any thickeners or storage tanks is defined.

- The pipeline route is defined (including any future extensions).

- The pipeline discharge condition, i.e. spigot discharge, open end or cyclones. The friction loss through spigot piping is higher than through straight piping and this additional friction loss must be considered in the system design.

\subsection{Tailings / paste properties}

The physical properties of the medium are defined:

- The solids density and particle size distribution.

- The medium $\mathrm{pH}$ and temperature range.

- Details of any flocculants or reagents to be added to the medium. 
- The medium rheology.

- The Miller Number. For positive displacement pump systems, this is used to establish the necessity of selecting piston diaphragm pumps instead of piston pumps.

- The corrosion rate to enable the pipeline wear allowance to be defined.

\subsection{Mechanical, piping, electrical and control and instrumentation}

These sections detail the following information:

- Mechanical: material, maximum pump operating speeds, sealing systems, etc.

- Piping: dimensional standards, steel grades, internal and external lining specifications, internal and external corrosion allowances, flanges or coupling specifications, and the pipeline support system.

- Electrical: outdoor and indoor design temperature and humidity, low and high voltage distribution and power, etc.

- Control and instrumentation standards for the project.

\subsection{Design codes and standards}

The design codes and standards applicable to the project are defined. Most high pressure systems are designed in accordance with ANSI/ASME Code for Pressure Piping B31.11, "Slurry Transportation Piping Systems".

\subsection{Costing basis}

For feasibility studies the following should be defined: study accuracy, costing battery limits, base date, currency and discount rate to be used for financial calculations.

\subsection{Key and unresolved issues}

All key design issues and decisions are documented. The document concludes with a list of unresolved issues - there should be no items on this list when the design is complete!

\section{TEST WORK}

\subsection{Representative sample}

A key issue for any test work campaign is obtaining representative samples. This is particularly difficult for green-field projects where limited quantities of ore are available for metallurgical pilot plant tests. The following points should also be considered when planning the test program: 
- Representative process water should be used for the test work as the water chemistry can have a significant effect on the tailings flow behaviour.

- For systems incorporating a thickener, the test work samples should be obtained from pilot sedimentation thickening using the proposed flocculent and dosage.

- If reagents are to be added to the tailings prior to deposition, the effect of the reagents should be investigated during the test campaign. Figure 2 shows the dramatic effect of lime addition on the rheology of a mineral sand tailings.

- For cemented pastes, the flow property tests should be performed using cemented tailings. Cement and binder from the intended source for the plant should be used for the tests.

- For pipeline design purposes, all flow behaviour measurements should be performed using fully sheared material.

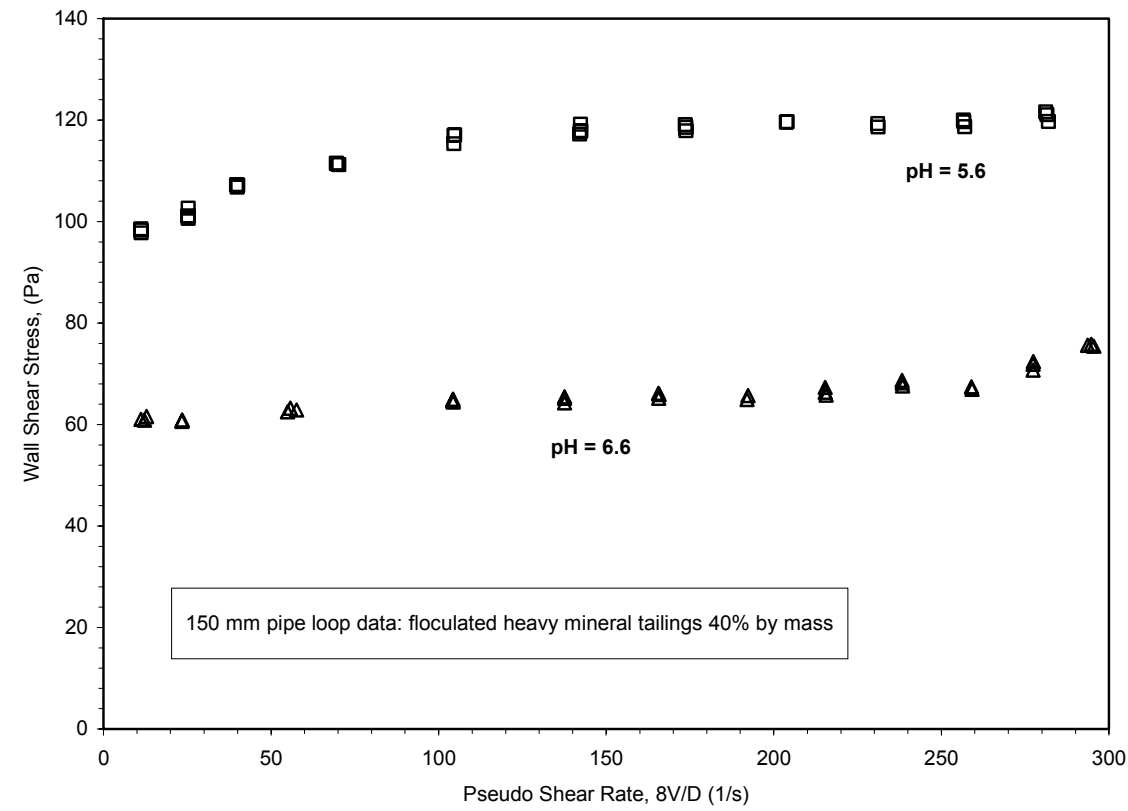

Figure 2 Effect of mixture pH on flow behaviour

\subsection{Material properties}

The solids specific gravity is measured using a standard test procedure such as ASTM D854-02.

There is no generally accepted method for determining the particle size distribution. The most commonly used methods are a combination of sieves and hydrometer (ASTM D422-63), and laser diffraction (e.g. Malvern particle size analyser). It is important to recognise that these methods do not give the same result due to the influence of particle shape on the measurement techniques. However, once a method has been adopted the results are consistent and comparisons can be made with previous data. 
Additional material property tests are:

- The freely settled concentration and the maximum packing concentration.

- The mixture $\mathrm{pH}$ should be measured for each test and ideally the water chemistry analysed.

- Tests to establish the mineral and chemical composition of the tailings are useful to aid in benchmarking the tailings with similar materials.

\subsection{Slump tests}

\subsubsection{Standard 12" slump cone}

A popular the method for obtaining an indication of flow behaviour is the standard 12" (305 $\mathrm{mm}$ tall) slump cone developed for concrete (ASTM C143/C 143M-00). Together with an empirical test data base, this test can be used to obtain a preliminary assessment of the paste pipeline flow behaviour for conceptual designs. However, the accuracy is not suitable for final design and specification of a pipeline system.

For cemented paste systems, the slump tests should be conducted with cement added to the paste. Figure 3 shows the effect of cement addition on the relationship between slump and mass concentration.

The standard slump cone is the industry bench mark in terms of communicating paste properties (e.g. $150 \mathrm{~mm}$ or 6" slump paste) and for paste plant quality control.

\subsubsection{Slump cylinder}

Pashais et al (1996) and Clayton et al (2003) have strongly advocated the use of the cylinder for slump tests in place of the standard slump cone. They note that the cylinder is cheaper to fabricate and more accurately predicts the mixture yield stress.

Based on our experience with the cylinder, we are not convinced that the derived yield stress from the measured slump is meaningful for pipeline flow conditions where the mixture is fully sheared. 


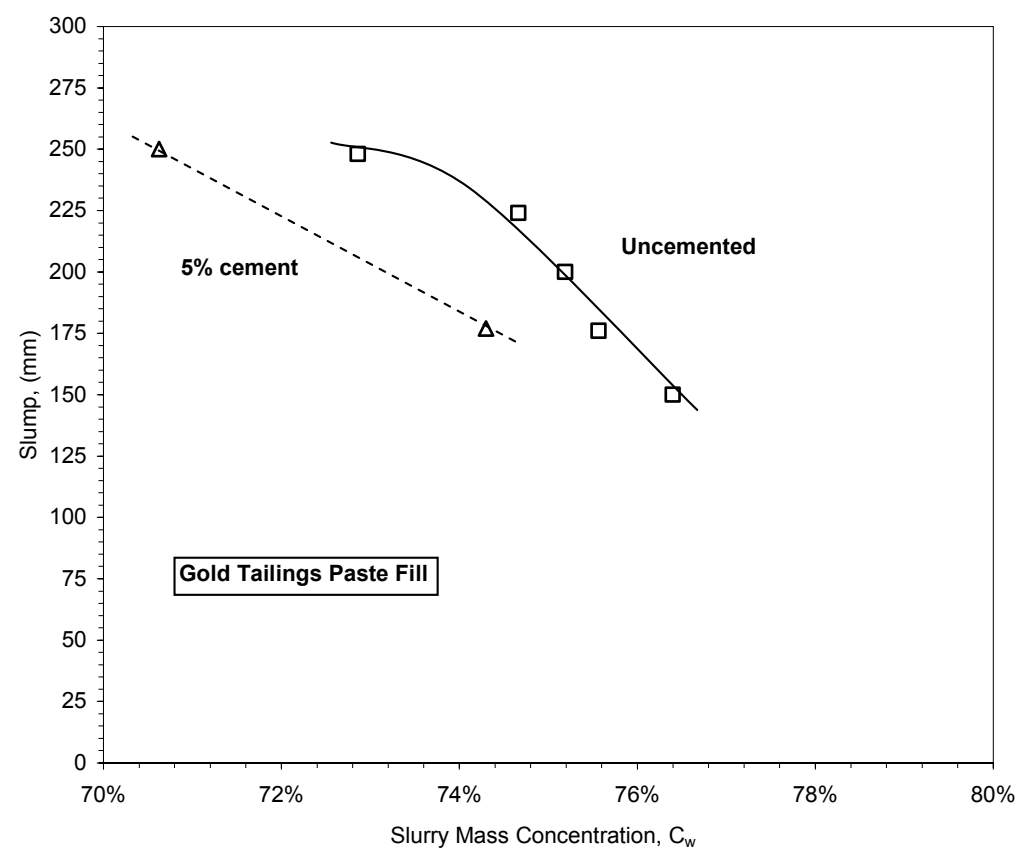

Figure 3 Effect of cement addition on slump (standard $300 \mathrm{~mm}$ slump cone)

\subsection{Rheology measurement}

\subsubsection{Rotational viscometers}

There are a number of proprietary rotational viscometers available for measuring rheology. A sample of the medium is placed in the annular gap between a stationary cup and a rotatable bob. Together with the system geometry, the rotational speed of the bob provides an indication of the shear rate and the torque required to rotate the bob provides an indication of the shear stress. Some researchers use a vane instead of the bob to measure the rheogram or flow curve (plot of shear stress verus shear rate for laminar flow conditions). Issues to be considered when conducting rotational viscometer tests are:

- The flow in the annular gap must be laminar without secondary flows or Taylor vortices.

- The medium rheology is required to determine the true shear rate for yield stress fluids, i.e. an iterative solution algorithm is required to determine the shear rate. This is often beyond the capability of the standard software provided with the instrument.

- The output must be checked for time or shear dependant effects (change in measured shear stress for constant bob speed and or non co-incident flow curves). The cause may be due to the nature of the medium or it may be due to particle effects caused by settling during test.

- The output must be checked for slip by conducting the tests in at least two geometries. The flow curves from the different geometries should be co-incident. If necessary, a slip correction procedure can be applied to the measured data. 


\subsubsection{Tube viscometers and pipe loop tests}

The distinction between tube (or capillary) viscometers and pipe loops is a little arbitrary as they are based on the same measurement principle:- the shear rate is determined from the flow rate though the tube and the shear stress from the pressure loss across the tube. For practical purposes, diameters up to $25 \mathrm{~mm}$ can be considered tube viscometers and larger diameters as pipe loops. Tube viscometers and pipe loops are favoured for pipeline design purposes due to the geometric similarity. The following issues should be considered:

- The output is in the form of a pseudo shear diagram (plot of pipe wall shear stress versus pseudo shear rate). The pseudo or bulk shear rate is defined as:

$$
\mathrm{T}=\frac{8 V_{m}}{D}
$$

where $V_{\mathrm{m}}=$ mean mixture velocity $(\mathrm{m} / \mathrm{s})$

$\mathrm{D}=$ internal pipe diameter $(\mathrm{m})$.

- For time independent Bingham Plastic mixtures, the pseudo shear rate is related to the rheology as follows (Govier and Aziz, 1972):

$$
\mathrm{T}=\frac{\tau_{\mathrm{o}}}{\mathrm{K}_{\mathrm{b}}}\left[1-\frac{4}{3}\left(\frac{\tau_{\mathrm{y}}}{\tau_{\mathrm{o}}}\right)+\frac{1}{3}\left(\frac{\tau_{\mathrm{y}}}{\tau_{\mathrm{o}}}\right)^{4}\right]
$$

where $\tau_{\mathrm{o}}=$ shear stress at pipe wall $(\mathrm{Pa})$

$\tau_{\mathrm{y}}=$ Bingham yield stress $(\mathrm{Pa})$

$\mathrm{K}_{\mathrm{b}}=$ Bingham fluid consistency index or plastic viscosity (Pa.s).

- Only laminar flow data should be considered as part of the pseudo shear diagram. Turbulent flow data is readily apparent from the differences in trends between laminar and turbulent data when plotted on a friction factor diagram.

- If required, the pseudo shear rate can be converted to the true shear rate using the RabinowitschMooney transformation (Govier and Aziz, 1972).

- The output must be checked for time or shear dependant effects. As with rotational viscometer tests, the cause may be due to the nature of the medium or it may be due to particle settling.

- The output must be checked for slip by conducting the tests in at least two pipe sizes. The pseudo shear diagrams should be co-incident. If necessary, a slip correction procedure may be applied.

- An adequate length of straight pipe must be provided before the pressure loss measurement section to ensure fully developed flow. Fifty pipe diameters is suggested for the approach length.

- Care must be taken to quantify particle degradation effects during closed loop pipe loop tests. This is particularly important when testing tailings-aggregate paste mixtures. 


\subsection{Wear and corrosion testing}

For positive displacement pump systems, the Miller Number testing (ASTM G75) should be conducted to determine sliding abrasion wear index values.

For unlined pipeline systems, the expected corrosion rate should be determined from corrosion tests (ASTM G31).

\section{FLOW BEHAVIOUR MODELLING}

\subsection{Laminar flow}

\subsubsection{Flow models}

The various laminar flow models used for thickened tailings and paste are discussed by Boger (2002). The model most commonly applied is the Bingham Plastic model:

$$
\tau=\tau_{\mathrm{y}}+\mathrm{K}_{\mathrm{b}} \dot{\gamma}
$$

where $\dot{\gamma}=$ shear rate $\left(\mathrm{s}^{-1}\right)$.

The above equation is fitted to the measured data to determine the rheological parameters. Equations 1 and 2 are solved to determine the wall shear stress for particular flow rate and pipe size.

\subsubsection{Pseudo shear diagram}

When working from the pseudo shear diagram, there is no need to identify a suitable rheological model or to establish the rheological parameters. For a particular pseudo shear rate, the wall shear stress can simply be determined directly from the pseudo shear diagram. Alternatively, the rheological parameters can be determined using an appropriate formula (e.g., Equation 2 for a Bingham Plastic mixture). Note that although the Bingham plastic model is a straight line on a rheogram, it is slightly curved on a pseudo shear diagram.

\subsection{Turbulent flow}

It is recommended that either the Wilson and Thomas (1985) or the Slatter (1999) models for turbulent flow of non-Newtonian mixtures are used to determine the turbulent flow pipeline wall shear stress.

\subsection{Operating flow regime}

Although the transition from laminar to turbulent flow occurs over a range of velocities, for design purposes it is generally considered to occur at a single velocity. A number of researchers have formulated various 
Reynolds numbers to predict this transition. In our experience, the most reliable technique is the intersection method:

- For the given conditions determine the pipeline wall shear stress for laminar and turbulent flow conditions as discussed above.

- The highest wall shear stress then dictates the operating flow regime.

Systems should not be designed to operate close to the laminar-turbulent transition due to the risk potential operational instabilities.

\subsection{Pipeline friction loss}

The pipeline friction pressure gradient is determined from the wall shear stress using the following equation:

$$
\frac{\Delta \mathrm{P}}{\Delta \mathrm{L}}=\frac{4 \tau_{\mathrm{o}}}{\mathrm{D}}
$$

\subsection{Pipe diameter selection}

Conventional tailings pipelines generally operate in turbulent flow and the pipe diameter is selected such that operating velocity is greater than the deposition velocity (the incipient velocity at which particles deposit on the pipe invert under turbulent flow conditions).

For paste and thickened tailings, different criteria apply. For laminar flow, the minium pressure gradient must be in the range of 1 and $2 \mathrm{kPa} / \mathrm{m}$ to avoid laminar flow settling (Cooke, 2002) as shown in Figure 4, or else the flow must be turbulent. For most thickened tailings applications, the velocity required to achieve turbulent flow is significantly higher than the deposition velocity.

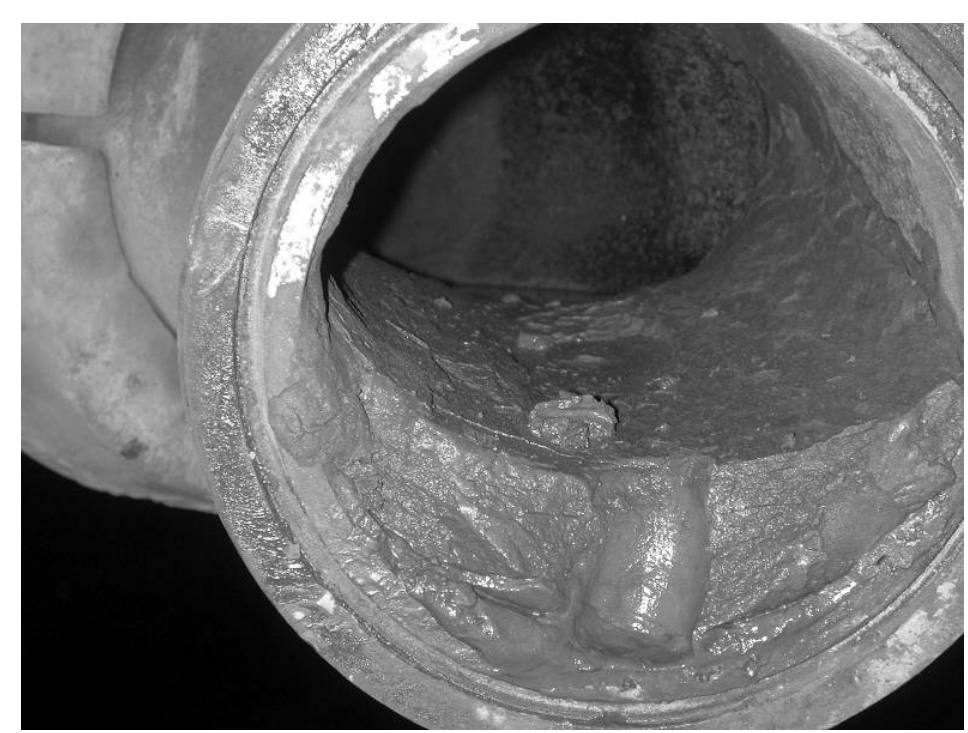

Figure $4 \quad$ Laminar flow settling in paste fill pipeline 


\section{ACKNOWLEDGEMENTS}

The author thanks Dr Paterson and Mr van Sittert for their comments and contributions to this paper.

\section{REFERENCES}

Boger, D.V. (2002) Paste and thickened tailings - a guide, Jewell, R.J., Fourie, A.B. and Lord, E.R. (eds), The Australian Centre for Geomechanics, Chapter 3.

Clayton, S., Grice, T.G. and Boger, D.V. (2003) Analysis of the slump test for on-site yield stress measurement of mineral suspensions. International Journal of Mineral Processing 70, pp. 3-21.

Cooke, R. (2002) Laminar flow settling: the potential for unexpected problems, Proc. 15th Int. Conf. on Hydrotransport, Bannf, Canada, 3-5 June.

Govier, G.W. and Aziz, K. (1972) The flow of complex mixtures in pipes, Van Nostrand Reinhold.

Pashias, N., Boger, D.V., Summers, J. and Glenister, D.J. (1996) A fifty cent rheomoter for yield stress measurement. Journal of Rheology 40(6), pp. 1179-1189.

Slatter, P.T. (1999) The role of rheology in the pipelining of mineral slurries, Min. Pro. Ext. Met. Rev., Vol 20, pp. 281-300.

Wilson, K.C. and Thomas, A.D. (1985) A new analysis of the turbulent flow of non-Newtonian fluids, The Canadian Journal of Chemical Engineering, 63, pp. 539-546. 